\title{
Research on Cascading Failures of Power Grid
}

\author{
Hongmin WANG ${ }^{1}$, Qiang WEI, Yaobin XIE \\ State Key Laboratory of Mathematical Engineering and Advanced Computing, \\ Zhengzhou 450001, China
}

\begin{abstract}
The safe and stable operation of power system is related to the national economy and people's livelihood of the whole country. Blackouts are almost always caused by cascading failures. This paper first analyzes the global blackouts in the past 20 years, and points out that the scale and probability distribution of blackouts obey the power-law relationship. Secondly, it is concluded that transmission line fault, bus fault, large-scale power flow transfer and relay protection device misoperation are the main factors causing cascading failures. Then, the five stages of cascading failure evolution are analyzed, which are slow successive interruption process, fast successive interruption process, transient oscillation, avalanche blackout and long recovery. Finally, the research results of cascading failure at home and abroad in recent years are analyzed from three research points: small world network, topological network and two-layer coupling network.
\end{abstract}

Keywords. Cascading failure, Cyber-physical system, Small world network, Twolayer coupling network

\section{Introduction}

As one of the most widely used artificial systems in the world, the normal operation of power system is related to the national economy and people's livelihood. With the continuous integration of power system, physical system and information system, smart grid is facing more and more attacks while improving its performance, such as False Data Injection Attack[1,2], DoS Attack[3,4], Replay Attack[5] and Route Interruption Attack[6]. Attackers often attack a single physical node in the network. The failure of a node in the network may affect the state and behavior of other nodes in the network, or even affect the whole network, resulting in cascading failure and paralysis of the whole network $[7,8]$. In order to ensure the safe and stable operation of national smart grid, researchers at home and abroad continue to optimize the cascading failure analysis. Therefore, cascading failure has always been a hot research topic[9-14].

In recent years, there have been many serious blackouts all over the world, almost all caused by cascading failures, which have a serious impact on the national economy and people's lives. Figure 1 lists the blackouts and their impacts in recent years.

It is found that before the occurrence of large-scale blackout, the power system usually operates in an unsafe and unstable state. The fault of power grid components triggers the system protection device, which causes the redistribution of power flow

${ }^{1}$ Corresponding Author, Hongmin WANG, State Key Laboratory of Mathematical Engineering and Advanced Computing, Zhengzhou 450001, China; Email: 1542811931@qq.com 
during the transfer of new operation state. In this process, the protection device is activated again, causing cascading accidents, leading to the occurrence of large-scale blackout[15]. At the same time, American researchers also attach great importance to the study of blackouts. Carreras, Newman and Dobson used the self-organized criticality theory to study the mechanism of blackouts. They analyzed the blackouts data of American power system in the past 15 years. The results show that the scale and probability distribution of blackouts obey the power-law relationship, which preliminarily proves that the American power grid has the characteristics of selforganized criticality[16]. The specific process of blackout is shown in figure 2 .

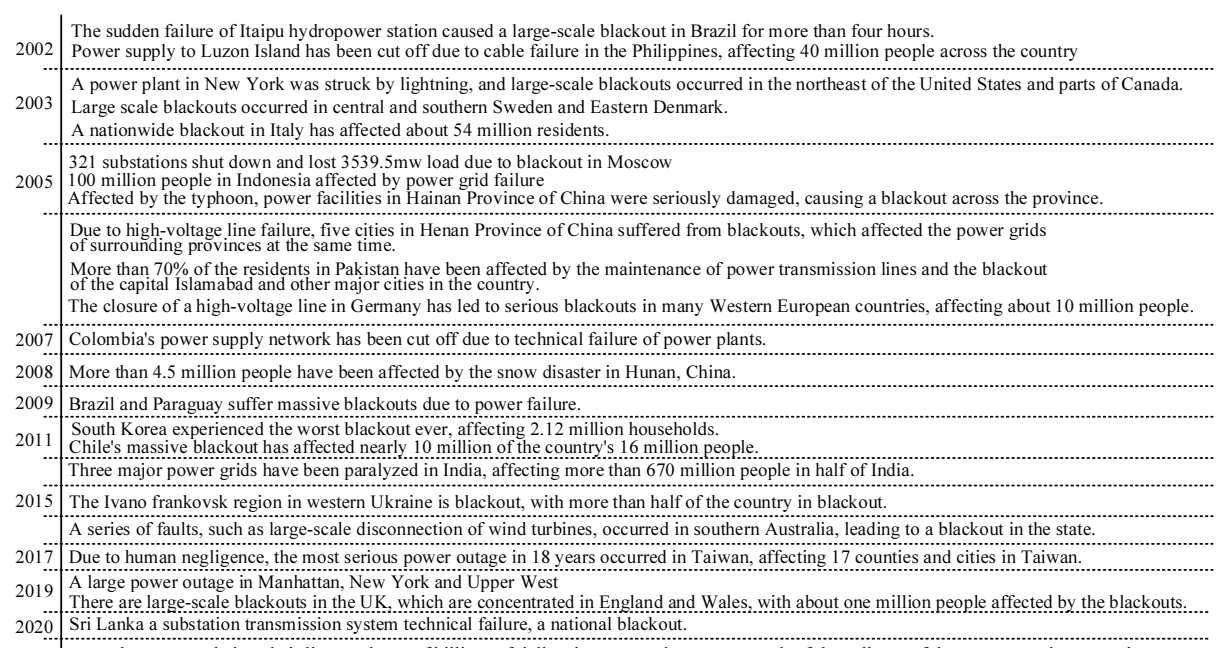

Figure 1. Blackouts and their impacts in the world in recent years

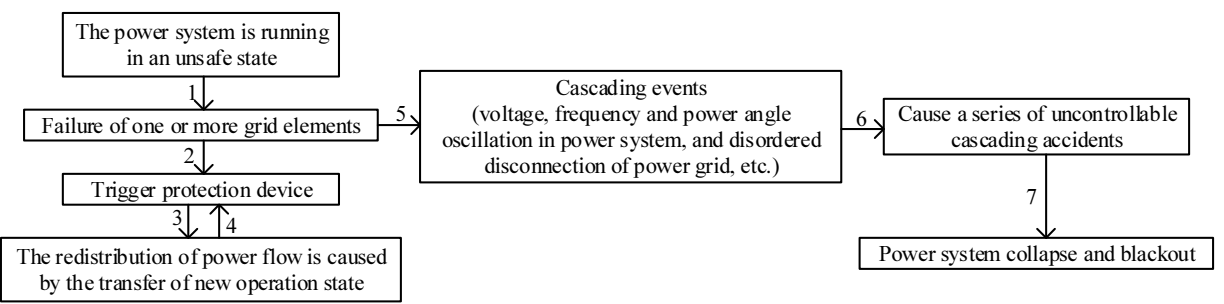

Figure 2. Flow chart of blackout

In fact, the main evolution form of blackout is cascading fault, which is characterized by the expansion from a single fault to multiple faults, and may even lead to the collapse of the whole power grid $[17,18]$. Once cascading faults occur in power system, it is very easy to cause large-scale blackouts. Therefore, the significance of cascading fault research is widely recognized by the power industry and academia. With the continuous strengthening of the interconnection and informatization of the power grid system, although there are various means of malicious attacks on the power grid, the ultimate cascading blackout must rely on the existing power infrastructure. Therefore, more and more people need to deeply understand the mechanism behind cascading failures in order to enhance the stability of power grid. 


\section{Analysis of Cascading Failure Factors}

Blackout has seriously affected the national economy and social life. So, it is urgent to adopt a more comprehensive and effective method to analyze the transmission characteristics of cascading failures in power grid, so as to provide a theoretical basis for the prevention and control of cascading failures in power system. When the grid failure occurs, the staff use scheduling to deal with the cascading failure, which can effectively reduce the impact of cascading failure.

\subsection{Failure Factors}

In order to effectively minimize the possibility of cascading failures, the main factors leading to cascading failures should be analyzed. It is found that there are four main causes of cascading failures, as shown in figure 3.

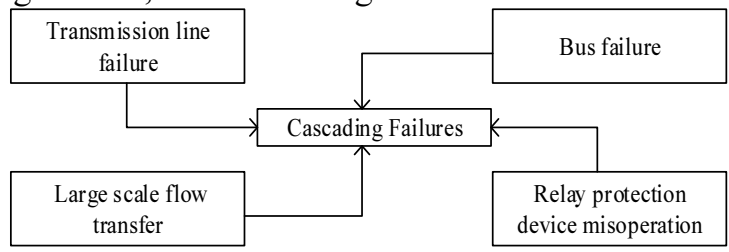

Figure 3. Main causes of cascading failures

(1) Transmission line failure

The causes of transmission line fault are lightning stroke, pollution flash, wind deviation, ice cover, bird damage, external force damage, etc. [19]. Transmission line failure will cause some units in the power plant to trip, which will cause the frequency of power equipment to drop rapidly. When the rapid decrease of frequency is effectively restrained by frequency response control, it will cause other units to trip, make the system frequency drop again and trigger the device of low frequency load shedding to participate in the work, cuts off some loads, triggers the protection misoperation, causing the large area paralysis of the power grid.

(2) Bus failure

The main reason for bus failure is air pollution and support insulator and bushing damage, which is less likely to occur, but it is very important for power grid operation[20]. Bus failure will lead to failure of all the components connected to it, destroy the stability of the whole power grid, and expand the accident, and bring serious consequences.

(3) Large scale flow transfer

Before the accident, due to various reasons, the line protection action quits operation, and then this part of the power flow which should be borne by the cut-off line is transferred to other tie lines in the network, resulting in the line overload quitting operation, further aggravating the severity of the power flow transfer of the system, thus causing a series of complex faults. Finally, due to the collapse of voltage and frequency, the system can not maintain normal operation state and is forced to split, resulting in a large area of power failure[21,22]. The schematic diagram of each stage of power flow transfer causing power outage is shown in figure 4. 


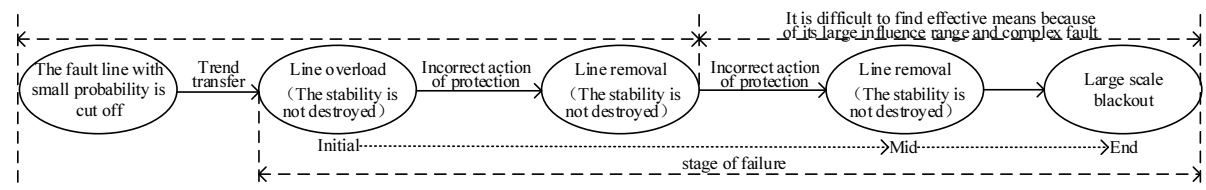

Figure 4. Phase diagram of blackout caused by power flow transfer

(4) Relay protection device misoperation

Relay protection aims to protect circuit parameters in normal range. On the one hand, when a single equipment of power system has fault or exceeds the permitted operation range, relay protection device quickly isolates it from other equipment of power system, so as to prevent other equipment from being affected and accident expansion, and to achieve timely stop loss. On the other hand, when the fault branch is generated due to relay protection tripping, it will also cause the change of the power system topology structure, and cause the redistribution of current in the power grid[23], which will cause the load in the cut-off line to be transferred to the remaining operation lines. The new distribution of current will cause the relay protection to cut off the line, thus leading to more lines exiting the operation, making the system run again, and reducing system stability. In short, the relay protection device is responsible for the fault propagation, and some misoperation will lead to the expansion of the fault range.

Through the analysis of cascade failure factors, the key characteristics of cascade faults can be found, which provides the breakthrough point for the prevention of blackout. If the power system produces the above four faults, in order to reduce the further spread of the fault, the relay protection device will quickly remove the fault elements. However, this may cause other lines in the system to overload or bus voltage deviate from the rated value seriously, and then trigger a new round of relay protection device action, and cause the next level fault[24]. Finally, it causes the failure diffusion and accident spread. In addition, the frequency of power equipment will change dramatically and substantially when the failure occurs, which is higher than or lower than the rated frequency threshold, which will destroy the stability of the whole system and break down the power system.

\subsection{Countermeasures -- Dispatching}

The way of dispatching blackout is very important to solve cascading failure effectively. For example, the blackout in Europe in 2006 was largely the result of human error. When the power system fails, the dispatcher will intervene to eliminate the fault. Due to the equipment and personal ability, the time of dispatching intervention in the system is not the same, which will cause different consequences. If the scheduling process is timely, the impact of cascading failures will be effectively reduced. For example, the scheduling results of the blackout in 2003 in the United States and Canada and the blackout in 2006 in Central China are compared as shown in table 1. 
Table 1. Comparison table of blackout dispatching treatment between America, Canada and central China

\begin{tabular}{l|l|l}
\hline Time & 2003-08-14 & 2006-07-01 \\
\hline Event & Blackout in USA and Canada & Blackout in Central China \\
\hline \multirow{2}{*}{$\begin{array}{l}\text { The condition assessment software } \\
\text { failed, and the dispatcher did not } \\
\text { take measures to eliminate the fault } \\
\text { action during this period. The } \\
\text { power outage lasted for 29 hours, } \\
\text { affecting the power consumption } \\
\text { of 50 million people in 8 states } \\
\text { (provinces) of the United States } \\
\text { and Canada. }\end{array}$} & $\begin{array}{l}\text { Two minutes after the initial } \\
\text { and adopts the strategy of machine } \\
\text { and load cut to maintain the } \\
\text { stability of the system. The final } \\
\text { outage time is 2 hours and 30 } \\
\text { minutes, which affects the power } \\
\text { consumption of some users in } \\
\text { Central China. }\end{array}$ \\
\hline
\end{tabular}

During the operation of the power system, the dispatcher of the power system monitors, commands and controls it to ensure the safe and economic operation of the power system and provide reliable and standard electric energy to users. Power system dispatching responsibilities are shown in figure 5.

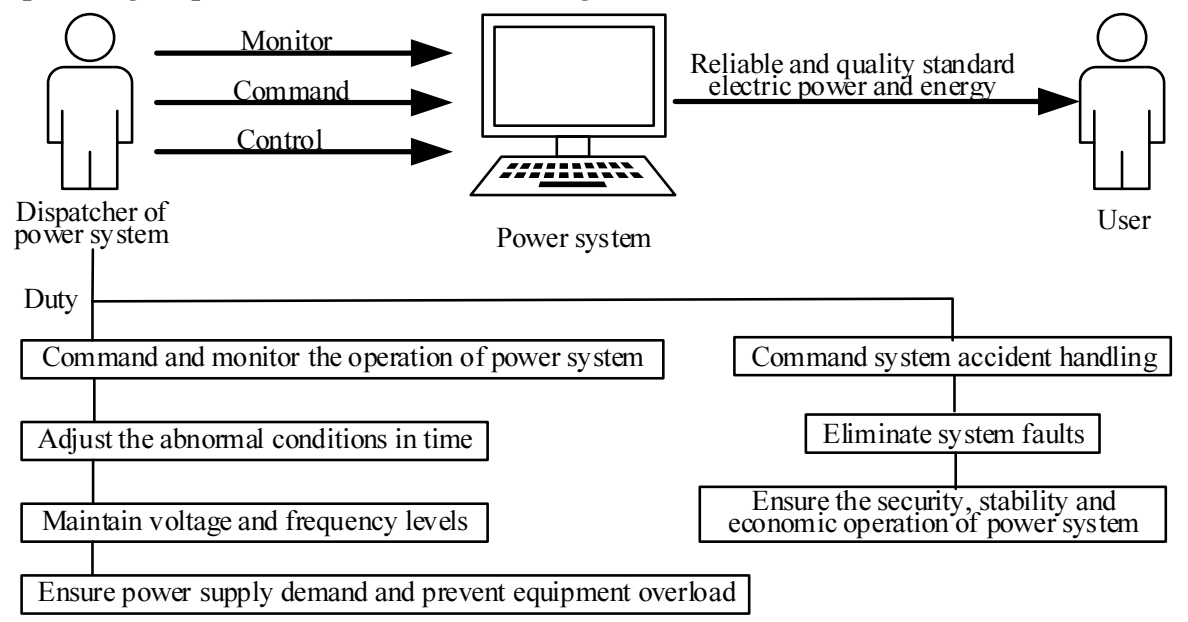

Figure 5. Power system dispatching responsibility chart

\section{Cascading Fault Development Process}

Cascading failure process is complex and has a unique propagation mechanism[12], and the causes of cascading failure are various. However, studies have shown that the development process of cascaded failures is mostly represented by the successive interruption of a series of components, which is mainly divided into five stages, including slow successive interruption process, fast successive interruption process, transient oscillation, avalanche blackout and long recovery process[13]. The development of cascading failures is shown in figure 6. 
Slow successive breaking $\rightarrow$ Fast successive breaking $\rightarrow$ A brief shock $\rightarrow$ Avalanche blackout $\rightarrow$ Slow recovery

Figure 6. Development process of cascading failures

The development process of the middle three is very short, and the dispatcher basically does not take any effective measures in this process, only takes corresponding measures in the slow successive stages[14]. The specific development process of blackout caused by cascading faults is shown in figure 7 .

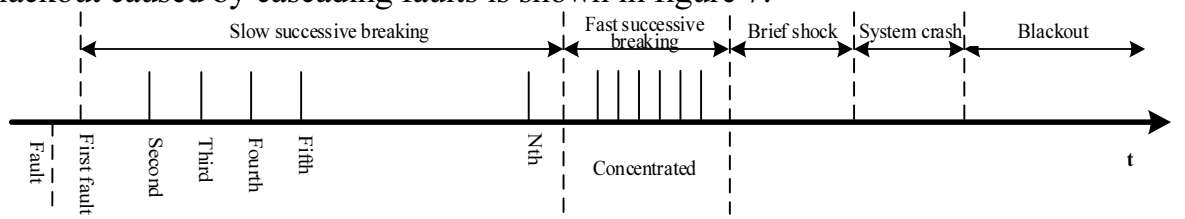

Figure 7. The diagram of development process of cascading blackout

At the slow continual outage stage, it usually meets the constraints of load and operation at the same time when the power system operates in a normal safe state. At this time, if the power system is attacked or accidental events occur, making it produce disturbance, the system can still meet the load constraints and operation constraints. At present, the correlation strength between various faults is relatively weak, which is called the correlation degree between faults. The calculation formula of correlation can be expressed by formula (1).

$$
\theta_{j}^{i}=\left\{\begin{array}{ll}
1 & i=j \\
\sum_{k=1}^{n} p_{i j k} w_{i j k} & i \neq j
\end{array} i, j=1,2,3, \ldots, m\right.
$$

Where $\theta_{j}^{i}$ is the degree to which node $J$ is affected by node $I$. The reason why the fault occurs is that the faults interact with each other, one node has a problem, which causes the power flow to move and causes the overload of the other node. Power flow transfer related mode, hidden fault related mode, network topology related mode and uncertain factor related mode are four typical inter link related modes[14]. Pijk is the related factor, which represents the impact of different related modes on $\theta_{\mathrm{j}}^{\mathrm{i}}$. Wijk is the weight of Pijk, and $\mathrm{M}$ is the number of nodes in the system.

The weak correlation between faults is generally expressed in the form of protection misoperation and circuit breaker refusing to operate. It may also be expressed as the droop of another transmission line due to heavy load caused by the outage of a line due to fault, which makes the following lines fail, such as the grounding fault in case of tree or ground flashover. This kind of weak correlation can also be reflected in the other two aspects: on the one hand, the period of the process is relatively long in the stage of slow successive interruption; on the other hand, the frequency of faults is usually low. With the successive occurrence of each fault event in the development process of cascaded faults, the correlation between faults is gradually enhanced. In this stage, if the dispatchers do not use timely and effective control measures, the system may enter the second stage of cascading fault development from the slow successive outage stage to the fast successive outage stage.

In the process of rapid successive interruptions, the power system is running in an emergency state. At this time, it will not be able to meet the operation constraints of the two constraints, and the system is on the verge of collapse. In this stage, the occurrence of fault events is more intensive, and the correlation between faults becomes stronger. The process of power flow transfer reflects the strong correlation between faults. Power 
flow transfer is easy to make other branches break, especially large-scale power flow transfer, which makes a series of lines trip due to overload, resulting in voltage and frequency fluctuations of the system and affecting its synchronous stability. Because of the relatively short time and short duration of the fast successive interruption phase, and the occurrence of more and more intensive faults, the frequency of faults is extremely high, which is another manifestation of the strong correlation between fault events.

With the continuous occurrence of faults, the lines of power system are broken one after another, and the robustness of the system is eroded and weakened a little bit. In the case of more and more frequent cascading interruptions, the system will deteriorate rapidly if the decision or prevention of the next possible interruption is not made in time. Most of the lines in the system are cut off one after another, which reduces the power transmission power to below the stability limit threshold, resulting in short-term oscillation of the power system, and then the collapse of the power system, and finally the formation of large-scale, large-scale blackout.

\section{Cascading Fault Analysis Method Based on Complex Network Theory}

In the mid-20th century, supervisory control and data acquisition (SCADA) began to be used in power grid[7], and power grid fault analysis technology was able to obtain data support. In the 1980s, expert system was applied to the analysis of power grid fault[8], which provided intelligent technical support for power grid fault analysis. As a result, relay protection management system (RPMS) was produced to monitor and analyze power grid fault. However, RPMS needs to build a dedicated data transmission channel for information collection, so wide area measurement system (WAMS) is gradually applied to the field of power grid fault diagnosis[25]. Various monitoring and analysis systems provide data and technical support for network fault analysis. In view of the global large-scale cascading blackout, researchers at home and abroad have studied its causes and mechanisms, and proposed a variety of cascading fault analysis methods. Among them, the cascading fault analysis method based on complex network theory is widely used in the research of related researchers.

The basic idea of complex network theory is to describe the skeleton structure of different systems in the form of network, and study the structural characteristics and dynamic behavior on the network[26]. According to the traditional way, complex network can be classified into the category of graph theory. Therefore, the research on cascading faults of power grid can be combined with many new concepts and methods proposed in the research of complex network. In recent years, some research results of power system cascading fault based on complex network theory are shown in table 2 .

Table 2. Some research results of cascading faults in power system based on complex network theory in recent years

\begin{tabular}{c|l|c}
\hline Research & \multicolumn{1}{c|}{ Description } & Reference \\
\hline $\begin{array}{c}\text { Small World } \\
\text { Network }\end{array}$ & $\begin{array}{l}\text { In power network graph, the distance between vertices } \\
\text { is relatively short, and the degree of connection between } \\
\text { them is close. }\end{array}$ & $\begin{array}{c}17 \sim 18, \\
27 \sim 33\end{array}$ \\
\hline $\begin{array}{c}\text { Topological } \\
\text { Network }\end{array}$ & $\begin{array}{l}\text { Combined with the electrical characteristics of power } \\
\text { grid, the robustness of power grid is studied by using } \\
\text { graph theory to control cascading fault propagation. }\end{array}$ & $34 \sim 45$ \\
\hline Two Layer & The power system is coupled with the information & $46 \sim 54$ \\
\hline
\end{tabular}


\begin{tabular}{l|l} 
Coupling & system, and the communication network and the power
\end{tabular}

Network supply network constitute a dependent network. The open circuit of the power system will directly lead to the termination of the power supply of the communication lines connected with it, and then lose its function.

\section{(1) Small world network}

Small world network is a kind of complex network with high information transmission efficiency, which has a great influence on complex networks[27,28]. Newman[17] pointed out that there are small world networks in the real world. The characteristic of small world networks is that the distance between the vertices in the network graph is short, and the clustering coefficient of the whole network is large, that is, the adjacent nodes of the vertices are closely connected with each other. In complex networks, the number of nodes in small world networks increases exponentially with the maximum value of the shortest path[29]. The characteristics of small world network are as follows:

$$
\left\{\begin{array}{l}
C \gg C_{\text {random }} \\
L \geq L_{\text {random }}
\end{array}\right.
$$

The Crandom is the clustering coefficient of the random network with the same number of nodes as the current network, and the Lrandom is the characteristic path length of the random network with the same number of edges as the current network.

The western power grid of the United States is defined as a typical small world network by Watts et al.[18] because of its small characteristic path length and high clustering coefficient, thus the complex network theory is introduced into the research of power network. In reference [30], the small world network model is discussed in depth. By comparing the structural characteristics of China's Northern Power Grid and the western power grid of the United States, it is pointed out that some regional networks in China are also small world networks. Reference [31] constructs a reasonable network topology model based on small world network, and proposes a method to predict the priority of cascading failures. Then, the weighted electrical betweenness and the energy index reflecting the change of power flow are constructed, and the cascading fault prediction model is proposed again. Reference [32] analyzes the vulnerability of power system in complex climate environment, and constructs a grid vulnerability analysis model based on climate weighted small world network interconnection. In reference [33], the propagation process of low-frequency oscillation is studied according to the small world characteristics of network. Low frequency oscillation is one of the causes of cascading failure. The low-frequency oscillation is studied through complex network theory. It is pointed out that the average path length determines the propagation speed of disturbance, and the clustering coefficient determines the size distribution of node phase oscillation. Most of the power grids in the world have the characteristics of small world. The destruction of key vertices and edges leads to the occurrence of network cascading failures.

(2) Topological network

Considering the classical circuit theory and the electrical characteristics of power grid[34-36], researchers pay attention to the electrical characteristics of each component, and make a detailed analysis of the power grid by using the electrical laws and the physical characteristics of the components. For example, in reference [36], considering the failure or misoperation of the protection equipment of power grid components, a 
cascading failure model caused by hidden fault tripping is established, which enhances the robustness of the power grid. Motter et al.[37] conducted a systematic study on the power network, using the knowledge of graph theory for reference, and pointed out that removing the heavy load vertices will cause the greatest damage to the network, and the network with more uniform vertex degree distribution is generally more robust. Crukitti et al.[38] analyzed the problem that the permanent deletion of network vertices is inconsistent with the actual situation, proposed a vertex recoverable use model, and confirmed that the heavily loaded vertices have decisive significance for the network topology. In reference [39], the relationship between topology and phase transition in power grid is analyzed, and the influence of topology on transition is evaluated by three spectral metrics. Chang et al.[40] studied the influence of key nodes or edges in topology on grid robustness. Reference [41] analyzes the relationship between the propagation rate of cascaded faults and the network topology parameters, and proves that the change of topology parameters is qualitatively consistent with the propagation rate of cascaded faults. In reference [42], in order to monitor the whole network, the SDN control plane is introduced into the communication network, which considers the topology area fault and optimizes the traffic redistribution strategy to reduce the impact of cascading fault. In reference [43], a new edge weight model based on node degree and betweenness is proposed to improve the invulnerability of topological networks. Literature [44] studies the impact of distribution network topology changes on wind turbine location and selection. Reference [45] proposed a node importance evaluation method based on the network dynamics model, combined with the network topology and the characteristics of the node itself, which made the evaluation results more comprehensive and further promoted the research of power grid robustness.

(3) Two layer coupling network

Buldyrev et al.[46] analyzed two or more power networks with certain dependence, and pointed out the influence of coupling on interdependent networks. Gao et al.[47] constructed a general analysis framework and studied the topological characteristics of multiple interdependent networks in detail. Bmmmitt et al.[48] studied the influence of inter system interdependencies on cascading failures of coupled networks, studied the sand pile model on the modular random graph and the graph based on actual graph, and pointed out that adding some network connections to the interdependent power grid can suppress the largest cascading in each system. Parshani et al.[49] studied the fault iterative cascading of two interdependent networks. When the key part of the node fails, the iterative process will lead to the transition of the penetration phase, leading to the failure of the two networks. It is also pointed out that reducing the coupling between networks will lead to the first-order penetration phase transition from the critical point to the second-order penetration phase transition, which verifies the jump of the cascading failure of power grid. Based on the control and monitoring of information system, Zhang Yin et al.[50] constructed a power grid cascading failure model considering the impact of information network damage, analyzed the impact of cascading failure caused by power grid crossing on the information network, and demonstrated that the ability of the system to suppress cascading failure of power grid is weakened with the loss of information network, resulting in the spread of cascading failure. Cordova garci et al.[51] studied the interdependence between power grid operation and communication control network, analyzed the impact of interdependence on cascading control, and proposed an effective cascading fault control algorithm for power grid. Reference [52] comprehensively evaluates and analyzes the physical information fusion system of power grid, studies the cascading accidents of power grid caused by the failure of 
monitoring function, and proposes a reliability evaluation method of power grid information system considering the role of information power. In reference [53], based on the cascading model of load, the restoration characteristics of power grid are studied for different coupling strength and network topology in interdependent networks, considering the maintenance resources, time sequence and load tolerance. Reference [54] explores the cascading fault propagation characteristics and robustness performance of smart grid coupled with power grid and communication network. Considering the physical characteristics and functions of nodes of power grid and communication network, and the influence of coupling relationship between different network layers, a two-layer network model of power grid communication network is proposed.

We can find that researchers try to build characteristic parameters in the simulation graph, such as load, capacity, and so on. They also design load redistribution for cascading fault propagation process, but this only helps us understand the cascading fault process, for the actual cascading fault The description of the process is totally wrong. The conclusion based on these models seems to be correct. However, we can infer from experience that the destruction of key vertices and edges will have a significant impact on the network. In the face of the increasingly large scale and complex topology of power grid, we need more accurate modeling of cascading faults.

\section{Summary}

As an indispensable resource in people's life, people are more and more dependent on the power system. Comprehensively promoting the construction of smart grid and cascading failure analysis has become an important part of national modernization and industrial intelligence construction. This paper studies the cascading failure of smart grid, analyzes the factors of cascading failure and the development process of cascading failure, and lists some fault analysis methods proposed by relevant researchers. However, most of the existing cascading fault analysis methods do not involve the problem of power angle stability, and lack of new power equipment and large-scale system to provide more reliable data.

\section{Reference}

[1] Wang Qi, Tai Wei, Tang Yi, Ni Ming. A Review on False Data Injection Attack Toward Cyber-physical Power System[J]. Acta Automatica Sinica, 2019, 45(1): 72-83.

[2] Zhao Junhua, Liang Gaoqi, Wen Fushuan, Dong Zhaoyang. Lessons Learnt from Ukrainian Blackout: Protecting Power Grids Against False Data Injection Attacks[J]. Automation of Electric Power Systems, 2016,40(07):149-151.

[3] Teixeira A, Sou K C, Sandberg H, et al. Secure control systems: A quantitative risk management approach[J]. IEEE Control Systems, 2015, 35(1): 24-45.

[4] Wang Yinan, Lin Yanjun, Li Huan, et al. Vulnerability analysis and countermeasures of electrical network control systems under DoS attacks[J]. Control and Decision, 2017, 32(03): 411-418.

[5] Teixeira A, Shames I, Sandberg H, et al. A secure control framework for resource-limited adversaries[J]. Automatica, 2015, 51: 135-148.

[6] Tang Yi, Chen Qian, Li Mengya, et al. Survey of network attack in power information physical fusion system environment[J]. Automation of Electric Power Systems, 2016,40(17):59-69.

[7] Russell J C, Masiell O R D, BOSE A. Power system control center concepts[C] // IEEE Power Industry Computer Applications Conference, May 15-18, 1979, Cleveland, USA.

[8] Fukui C, Kawakami J. An expert system for fault section estimation using information from protective relays and circuit breakers[J]. IEEE Trans on Power Delivery, 1986, 1(4): 83-90. 
[9] Wang Ming. Research on the Identification Method of Vulnerable Parts of Power Grid Considering Attack Risk[D]. Chongqing University,2016.

[10] Meng Haijun. Statistics laws of great blackouts at home and abroad and emergency disposal system assessment[D]. North China Electric Power University,2008.

[11] Du Qizhuang. Research on Under Frequency Load Shedding Based on Long-term Stability of Power System[D]. North China Electric Power University,2006.

[12] Cai Ye, Cao Yijia, Tan Yudong, et al. Influences of Power Grid Structure on Cascading Failure Based on Standard Structure Entropy[J]. Transactions of China Electrotechnical Society, 2015, 30(03):36-43.

[13] Xue Yusheng. Space-time Cooperative Framework for Defending Blackouts Part I From Isolated Defense Lines to Coordinated Defending[J]. Automation of Electric Power Systems,2006(01):8-16.

[14] Xiao Xian, Zhou Buxiang, Liu Nian, et al. Related Model Used to Simulate Slow Break Stage of Power Grid Cascaded Failure[J]. Proceedings of the CSU-EPSA,2015,27(03):76-80,86.

[15] Zhu Ziqiang. Impact of Protection system on the Development of Power System Cascading Failures[J]. Hefei University of Technology, 2015.

[16] B. A. Carreras, Poole A B, Newman D E, et al. Initial Evidence for Self-Organized Criticality in Electric Power System Blackouts[C] // Hawaii International Conference on System Sciences. Hicss. IEEE Computer Society, 2001:705-709.

[17] Newman M. E. J. The Structure and Function of Complex Networks[J]. SIAM Review,2003,45(2): 167256.

[18] Duncan J. Watts, Steven H. Strogatz. Collective dynamics of 'small-world' networks[J]. Nature: International weekly journal of science,1998,393(6684): 440-442.

[19] Wang Jian, Xiong Xiaofu, Li Zhe, et al. Time distribution of weather-related transmission line failure and its fitting[J]. Electric Power Automation Equipment,2016,36(03):109-114+123.

[20] Lu Zhifeng, Zhou Jiaqi. Reliability evaluation of distribution system considering faults occurred in switches and buses[J]. Power System Technology,2002(04):26-29.

[21] Luo Bin, Li Gang, Miao Shumin, et al. A Parallel Batch Method for Power Flow Transferring Analysis in Large-scale Power Systems[J]. Proceedings of the CSEE,2015,35(21):5446-5454.

[22] He Peicheng. Study of Emergency Control Strategy to Prevent Cascading Trip Caused by Power Flow Transferring[D]. North China Electric Power University,2017.

[23] Grigoraș G, Neagu B C, Scarlatache F, et al. Bi-Level Phase Load Balancing Methodology with Clustering-Based Consumers' Selection Criterion for Switching Device Placement in Low Voltage Distribution Networks[J]. Mathematics,2021,9(5).

[24] Yang Shenhao, Chen Weirong, Zhang Xuexia, et al. A Graph-based Method for Vulnerability Analysis of Renewable Energy integrated Power Systems to Cascading Failures[J]. Reliability Engineering \& System Safety,2021,207.

[25] WANG C, DOU C X, LI X B, et al. A WAMS/PMU-based fault location technique[J]. Electric Power Systems Research, 2007, 77(8): 936-945.

[26] Hu Juan. Research on Complex Network Theory and Its Application in Electric Power System[D]. Huazhong University of Science and Technology,2008.

[27] Li Shenghu, Ding Ming, Wang Min, et al. Search of power system chained failure mode considering uncertainty of element fault and performance of protective relaying[J]. Power System Technology, 2004(13):27-31+44.

[28] Zhao Xin. Small-world Network Theory and its Application in Wind Power Short-term Forecasting[D]. Beijing Jiaotong University, 2015.

[29] Chen Zhipeng, Xie Ning, Wang Chengmin, et al. Vulnerability Evaluation and Robustness Improvement Strategy of Complex Power Network Based on Fractal Mechanism[J]. Power System Technology, 2021,45(02):657-665.

[30] Meng Zhongrwei, Lu Zongriang, Song Jingyan. Comparison analysis of the small-world topological model of Chinese an American power grids[J]. Automation of Electric Power Systems, 2004(15):21$24+29$.

[31] Pan Yifen, Li Huaqiang, He Hanfeng, et al. Cascading Failures Prediction of 'Transmission Network in Small World Network[J]. Proceedings of the CSU-EPSA, 2013,25(02):148-153.

[32] Sun Xiaowei, Li Peng, Sun Liqiang, et al. Vulnerability Analysis Model of Power System Based on Meteorological Weighted Small-world Network[J]. Control Engineering of China, 2020,27(12):21432150.

[33] Wu Jinjie. Research on propagation characteristics of low frequency oscillation based on small-world network[D]. Harbin Institute of Technology,2019.

[34] Liu Y, Xue Y S. Cascading Outage Analyses by Integrating Distribution Factor Method with AC Power Flow[J]. IEEE Access, 2019, 7: 180887-180897.

[35] Jun Yan, Tang Yufei, HE Haibo, et al. Cascading Failure Analysis with DC Power Flow Model and Transient Stability Analysis[J]. IEEE Transactions on Power Systems, 2015, 30(1):285-297. 
[36] Garlapati S, Hua Lin, Sambamoorthy S, et al. Agent Based Supervision of Zone 3 Relays to Prevent Hidden Failure Based Tripping[C]//Proceedings of IEEE International Conference on Smart Grid Communications. Washington D. C., USA: IEEE Press, 2010: 256-261.

[37] Motter Adilson E, Lai Ying-Cheng. Cascade-based attacks on complex networks.[J]. Physical review. E, Statistical, nonlinear, and soft matter physics,2002,66(6 Pt 2): 65-102.

[38] Crucitti Paolo, Latora Vito, Marchiori Massimo. Model for cascading failures in complex networks.[J]. Physical review. E, Statistical, nonlinear, and soft matter physics,2004,69(4 Pt 2): 45-104.

[39] Koç Y, Warnier M, Van Mieghem P, et al. A topological investigation of phase transitions of cascading failures in power grids[J]. Physica A: Statistical Mechanics and its Applications, 2014, 415: 273-284.

[40] Chang L, Wu Z. Performance and reliability of electrical power grids under cascading failures[J]. International Journal of Electrical Power \& Energy Systems, 2011, 33(8): 1410-1419.

[41] Dey P, Mehra R, Kazi F, et al. Impact of Topology on the Propagation of Cascading Failure in Power Grid [J]. IEEE Transactions on Smart Grid, 2016, 7(4): 1970-1978.

[42] Lanlan R, Xiaomei W, Xuesong Q. Analysis of Cascade Fault Optimization Based on Regional Fault and Traffic Reallocation in Complex Networks[J]. IEEE Access, 2018, 6: 24818-24828.

[43] Cui Wenyan, Meng Xiangru, Kang Qiaoyan, et al. Optimization of cascading invulnerability on weighted complex networks based on composite edge weight model[J]. Systems Engineering and Electronics, 2017,39(2):355-361.

[44] Weerasinghe D J, Pahwa S, Miller R D, et al. Impact of Intentional Structural Changes in Distribution System Topology to Wind Turbine Siting and Sizing: A Complex Network Approach[J]. Nephron Clinical Practice, 2015, 2(1): -.

[45] Kong Jiangtao, Huang Jian, Gong Jianxing, et al. Evaluation methods of node importance in undirected weighted networks based on complex network dynamics models[J]. Acta Phys. Sin., 2018,67(09):255271.

[46] Buldyrev Sergey V, Parshani Roni, Paul Gerald, Stanley H Eugene, Havlin Shlomo. Catastrophic cascade of failures in interdependent networks[J]. Nature,2010,464(7291): 1025-1028.

[47] Gao J, Buldyrev S V, Havlin S, et al. Robustness of a network of networks[J]. Physical Review Letters, 2011, 107(19): 195701.

[48] Brummitt C D, D' Souza R M, Leicht E A. Suppressing cascades of load in interdependent networks[J]. Proceedings of the National Academy of Sciences, 2012 109(12): E680-E689.

[49] Parshani R, Buldyrev S V, Havlin S. Interdependent networks: Reducing the coupling strength leads to a change from a first to second order percolation transition[J]. Physical Review Letteres, 2010, 105(4): 048701.

[50] Zhang Yin, Xiao Xianyong, Chen Jing, et al. Impact of Information Network Damage on Overload-based Cascading Failures of Power Grid[J]. Automation of Electric Power Systems, 2017,41(13): 14-21.

[51] Cordova-Garcia J, Wang X, Xie D, et al. Control of communications-dependent cascading failures in power grids[J]. IEEE Transactions on Smart Grid, 2019, 10(5): 5021-5031.

[52] Guo Jia. Research on Cyber-Physical Power System (CPPS) Vulnerability Assessment Methods Considering Cascading Failures[D]. Zhejiang University, 2017.

[53] Zhong Jilong, Zhang FengMing, Yang Shunkun, Li Daqing. Restoration of interdependent network against cascading overload failure[J]. Physica A: Statistical Mechanics and its Applications,2019,514.

[54] Wang Wenbo. Research on cascading failure modeling and robustness of power grids based on complex networks[D]. Nanjing University of Posts and Telecommunications, 2020 